\title{
OPTICAL PROPERTIES OF MOLECULAR BEAM EPITAXY GROWN ZnSe ON GaAs
}

\author{
K. Karpińska, A. SUChocki, M. GodLEwski \\ Institute of Physics, Polish Academy of Sciences \\ Al. Lotników 32/46, 02-668 Warszawa, Poland \\ AND D. HoMmeL
}

Physikalisches Institut der Universität Würzburg Am Hubland, 8700 Würzburg, Germany

\begin{abstract}
Photoluminescence studies of molecular beam epitaxy grown ZnSe-on-GaAs layers are presented. The high sensitivity of the PL technique allowed for identification unintentional dopants in pure $\mathrm{ZnSe}$ sample. Characteristic photoluminescence lines due to extended defects were observed. The experimental results obtained show a correlation between intentional doping level and extended defects concentration. We conclude also that even though molecular beam epitaxy layers are grown at low temperature, the self-compensation mechanism may still be important. For heavily doped sample edge emission is deactivated likely due to efficient energy transfer link with deep donor-acceptor pair bands.
\end{abstract}

PACS numbers: 68.55.Bd, 68.55.Ln, 78.66.Fd

\section{Introduction}

Molecular beam epitaxy (MBE) grown $\mathrm{ZnSe}$ on $\langle 100\rangle$ GaAs substrate belongs to the most promising material for constructing blue emitting laser diodes [1]. MBE layers studied in the present work are prepared for such an application. Photoluminescence (PL) studies reported here were performed' in order to characterize material properties of either undoped or chlorine doped ZnSe MBE epilayers. The aim of these studies was to control contamination level, to correlate extended defects concentration with intentional doping level and to verify the role of selfcompensation processes [2] in MBE grown material. 


\section{Experimental}

The ZnSe samples studied were grown on $\langle 100\rangle$ GaAs substrate by the MBE method in a Riber 2300 growth chamber. Three different $1 \mu \mathrm{m}$ thick epilayers were examined. \#ZS-26 sample was undoped. Two others were $n$-type chlorine doped using $\mathrm{ZnCl}_{2}$ as source material. \#ZS-n 14 was highly doped with room temperature free electrons concentration in mid $10^{18} \mathrm{~cm}^{-3}$. \#ZS-n 16 sample was lightly doped with free electrons concentration about $8 \times 10^{15} \mathrm{~cm}^{-3}$.

Photoluminescence spectrum was excited with uv multiline of the Inova 200 argon laser, dispersed with the GDM 1000 double grating monochromator, and was detected with liquid nitrogen cooled S1 EMI photomultiplier and the Stanford Research SR530 lock-in amplifier. The samples were mounted in Leybold closed cycle cryogenic refrigerator.

\section{Photolumincscence studies}

The high resolution PL study of three epilayers is shown in Fig. 1. For \#ZS-26 epilayer the PL spectrum is dominated by the free excitonic (FE) emission at $2.804 \mathrm{eV}[3]$ and by the so-called $Y$ PL band $(2.604 \mathrm{eV})$, which previously was attributed to the recombination at dislocations [4]. A weak dislocation related

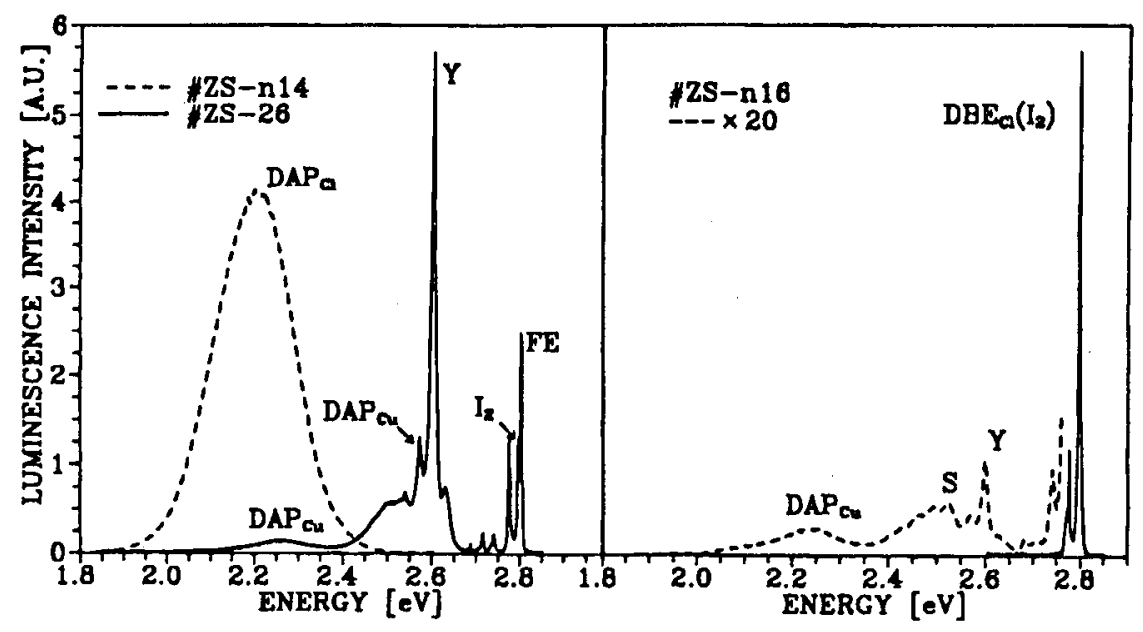

Fig. 1. The photoluminescence spectrum of three MBE grown ZnSe samples. PL spectrum was recorded at $8 \mathrm{~K}$ temperature under uv line excitation of argon laser. The explanation of the origin of different PL bands is given in the text.

PL at $2.74 \mathrm{eV}$ is also observed [5]. The large strength of the $Y$ band means that lattice-mismatch strain is relaxed by formation of misfit dislocations [6]. We notice also that the strain present in the epilayer shifts some PL bands by a few $\mathrm{meV}$ from their position in bulk ZnSe. The FE PL was observed up to $200 \mathrm{~K}$. This was the most stable component of the edge emission. The dominating $Y$ band rapidly disappears at increased temperatures and is not observed for above $100 \mathrm{~K}$. 
The high resolution PL measurements allowed us to identify unintentional dopants $(\mathrm{Cl}, \mathrm{Na}$ and $\mathrm{Cu})$ present in the undoped $\mathrm{ZnSe}$ sample. This we conclude from the observation of weak PL bands attributed previously to neutral $\mathrm{Cl}$ donor bound exciton (DBE) recombination $\left(I_{2} \mathrm{PL}\right.$ at $2.797 \mathrm{eV}$ [7]) and donor-acceptor pair (DAP) transitions for $\mathrm{Na}(2.715 \mathrm{eV}[8])$ and $\mathrm{Cu}(2.631 \mathrm{eV}$ [9] and $2.256 \mathrm{eV}$ [10]) acceptors. Contaminant concentration is low, as concluded from relatively ligh intensity of the FE line. The identity of $2.777 \mathrm{eV} \mathrm{PL}$ is not known.

For lightly doped \#ZS-n 16 sample the PL spectrum is dominated by the Cl-related $I_{2} \mathrm{DBE}$ emission. We report also the appearance of the so-called $S$ band at $2.519 \mathrm{eV}$, assigned to the shallow donor-deep acceptor transition $[5,11]$, with its LO phonon replicas. The $Y$ band is much weaker and becomes comparable in the intensity to the $2.740 \mathrm{eV}$ PL also related to some extended defect. The change of the relative intensity of these two bands is not understood at present.

For the heavily $\mathrm{Cl}$ doped \#ZS-n 14 sample the edge emissions are very weak and the PL spectrum is dominated by the broad yellow-green emission of the donor-acceptor pair nature [12]. The heavily doped sample shows some spatial inhomogeneity and depending on the excitation spot either Cl-related self-activated (2.0327 eV [13]) or $2.206 \mathrm{eV} \mathrm{DAP}$ emissions dominate. Figure 2 shows the temperature dependence of the latter PL band. It decreases in intensity at increased

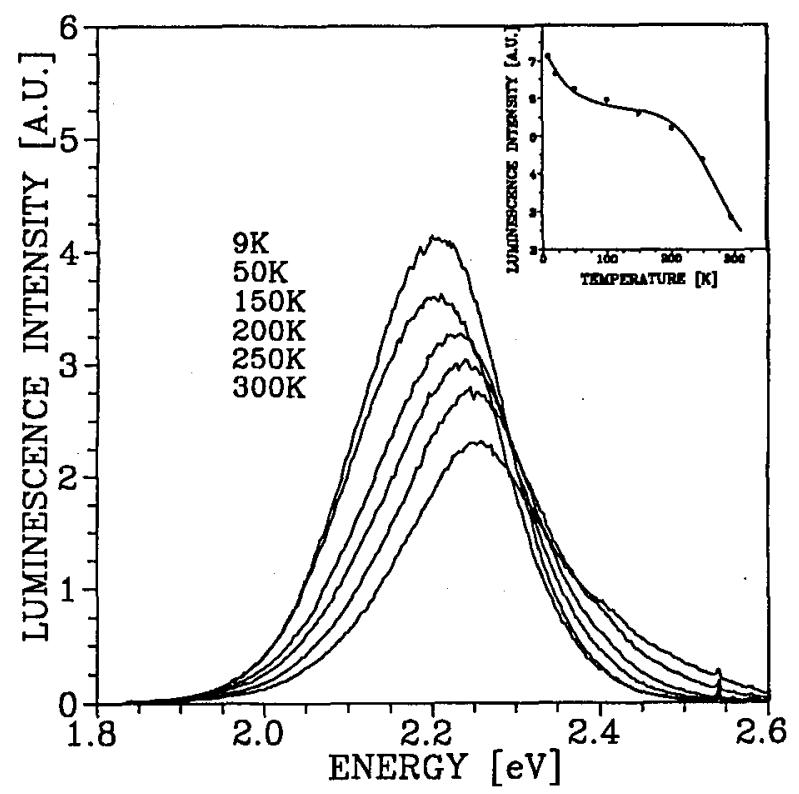

Fig. 2. The temperature dependence of the broad emission band at $2.206 \mathrm{eV}$ is observed in the heavily $\mathrm{Cl}$ doped \#ZS-n 14 epilayer. For increased temperatures the Cu-related $\mathrm{DAP}$ band at $2.256 \mathrm{eV}$ starts to dominate.

temperatures with the same activation energy as observed for the edge emissions for a lightly doped sample. The deactivation energy equals the exciton binding 
energy in $\mathrm{ZnSe}$. This result indicates an efficient energy transfer link between edge excitonic emissions and a deep DAP transition. We expect that a relative weakness of the edge PL may be explained in this way. For room temperature Cu-related band at $2.256 \mathrm{eV}$ dominates. The broad PL band is thus of complex nature and is due to at least two overlapping DAP transitions.

The PL bands connected with recombination at extended defects are missing. This suggests that intentionally introduced $\mathrm{Cl}$ decorates dislocations.

\section{References}

[1] M.A. Haase, J. Qin, J.M. DePuydt, M. Cheng, Appl. Phys. Lett. 59, 1272 (1991).

[2] Y. Marfaing, Prog. Crystal Growth Charact. 4, 317 (1981).

[3] H. Venghaus, Phys. Rev. B 19, 3071 (1979).

[4] J. Gutowski, N. Presser, G. Kudlek, Phys. Status Solidi A 120, 11 (1990).

[5] P.J. Dean, Phys. Status Solidi A 81, 625 (1984).

[6] J. Gutowski, Semicond. Sci. Technol. 6, A51 (1991).

[7] J.L. Merz, H. Kukimoto, K. Nassau, J.W. Shiever, Phys. Rev. B 6, 545 (1972).

[8] V. Swaminathan, L.C. Greene, Phys. Rev. B 14, 5351 (1976).

[9] G. Jones, J. Woods, J. Lumin. 9, 389 (1974).

[10] P.J. Dean, Prog. Solid State Chem. 8, 1 (1973).

[11] K.L. Lewis, D.J. Cook, P.B. Roscoe, J. Cryst. Growth 56, 614 (1982).

[12] M. Godlewski, W.E. Lamb, B.C. Cavenett, Solid State Commun. 39, 595 (1981).

[13] S. Tida, J. Phys. Soc. Jpn. 25, 177 (1968). 